\title{
KLF6: Mutational analysis and effect on cancer cell proliferation
}

\author{
DONG YIN $^{1 *}$, NAOKI KOMATSU ${ }^{1,3 *}$, CARL W. MILLER ${ }^{1}$, ALEXEY M. CHUMAKOV ${ }^{1}$, \\ ALBERTO MARSCHESKY $^{4}$, ROBERT McKENNA ${ }^{5}$, KEITH L. BLACK ${ }^{2}$ and H. PHILLIP KOEFFLER ${ }^{1}$ \\ ${ }^{1}$ Division of Hematology/Oncology, Cedars-Sinai Medical Center/University of California at Los Angeles \\ School of Medicine; ${ }^{2}$ Maxine Dunitz Neurosurgical Institute, Cedars-Sinai Medical Center, UCLA School of \\ Medicine, Los Angeles, CA, USA; ${ }^{3}$ Department of Hematology/Respiratory, Kochi Medical School, Okohcho, \\ Nankoku, Kochi, Japan; ${ }^{4}$ Departments of Pathology, Cedars-Sinai Medical Center/University of California \\ at Los Angeles School of Medicine; ${ }^{5}$ Department of Surgery, Cedars-Sinai Medical Center/ \\ University of California at Los Angeles School of Medicine, Los Angeles, CA, USA
}

Received June 7, 2006; Accepted August 7, 2006

\begin{abstract}
Kruppel-like factor 6 (KLF6/Zf9/CPBP), a member of the Kruppel-like family of zinc finger transcription factors, has recently been suggested to be a mutated tumor suppressor in selected human cancers. Initially, we investigated whether the KLF6 gene was altered in 36 paired non-small cell lung cancers (NSCLC), 89 brain tumors, 7 normal brains, 46 cancer cell lines from a large variety of tissues, and 144 peripheral blood cells from healthy individuals using single strand conformation polymorphism (PCR-SSCP) and DNA sequencing. Changes in the coding region of KLF6 were found in brain tumors (missense changes, $8 \%$; silent polymorphisms, $2 \%$ ), lung cancers (missense changes, $3 \%$; silent polymorphisms, $6 \%$ ) and cancer cell lines (missense changes, 2\%; silent polymorphisms, 2\%). All of the nucleotide changes in the lung tumor samples were present in their matched normal samples, suggesting that these changes were germline polymorphism. Many of the altered KLF6 genes found in the brain tumors were cloned into an expression vector and placed into a GBM cell line, and cell growth was monitored. Wild-type, deleted exon 3, or E30G missense KLF6 significantly reduced cell growth; in contrast, forced expression of KLF6 having either the S92R, P183L or A276G missense substitution did not alter the growth of transfected GBM cells $(\mathrm{p}>0.05)$. Expression levels of KLF6 were higher in normal brain samples than in glioma samples as measured by real-time RT-PCR $(\mathrm{p}<0.05)$.
\end{abstract}

Correspondence to: Dr Dong Yin, Division of Hematology and Oncology, Cedars-Sinai Medical Center, UCLA School of Medicine, 8700 Beverly Boulevard, Davis Building, Room 5022, Los Angeles, CA 90048, USA

E-mail: dong.yin@cshs.org

${ }^{*}$ Contributed equally

Key words: Kruppel-like family zinc finger protein, point mutation, brain tumor, lung cancer
To our surprise, nucleotide changes were found at $-4,-5$, and -6 upstream of the start of translation in $45 \%$ of brain tumors, and $10 \%$ of normal blood samples. Focusing on the most frequent alteration $(-4 \mathrm{C}>\mathrm{A})$, the nucleotide change did not affect translation of KLF6. Taking together, KLF6 coding sequences are altered in $10 \%$ brain tumors, $8 \%$ NSLC, and $4 \%$ of cancer cell lines. All of those observed in lung cancer are germline polymorphisms. Several additional ones identified in GBM, have lost their ability to slow the growth of glioma cells; furthermore, a proportion of GBM have decreased expression of KLF6 as compared to normal brain tissue. Dysfunction of this gene may contribute to oncogenesis in the brain.

\section{Introduction}

Recent studies suggest that Kruppel-like factor 6 (KLF6), which is also called Zinc finger transcription factor Zf9 (Zf9) or core promoter element binding protein (CPBP or COPEB), may be an altered tumor suppressor in some human cancers. Loss of heterozygosity $(\mathrm{LOH})$ in the region of this gene was reported in $77 \%$ of prostate cancers, with mutations of $K L F 6$ found in $71 \%$ of these cases (1). These investigators found that forced overexpression of wild-type KLF6 up-regulated $\mathrm{p} 21^{\text {waf } 1}$ associated with suppression of growth of prostate cancer cells in a p53-independent manner. Another study found missense mutations of KLF6 in 16\% (3/19) of sporadic nasopharyngeal carcinomas (2). In addition, investigators have reported mutations or inactivation of KLF6 in gliomas (3), pituitary tumors (4), non-small cell lung cancers (5), and colorectal cancers (6). In contrast, several other large studies of prostate cancer (7), hepatocellular carcinomas (8) and colorectal cancers (9) did not find mutations, but did find frequent polymorphisms.

KLF6 is a ubiquitously expressed Kruppel-like transcription factor, whose function is unresolved. The gene is mapped near the telomere of chromosome 10p (10p15.2), and consists of 4 exons, encoding a nuclear core promoter element binding protein. This protein has 3 zinc fingers at its 
C-terminal end, a serine/threonine-rich central region and an acidic domain lying within the $\mathrm{N}$-terminal region. The zinc fingers of this protein are responsible for specific DNA binding to the guanine-rich core promoter elements. The central region may be involved in either activation or posttranslational regulatory pathways, and the acidic N-terminal domain contains the transcriptional activation motif. Putative transcriptional targets of KLF6 include the genes encoding placental glycoprotein (10), collagen $\alpha 1$ (11), transforming growth factor $\beta 1$ (TGFß1), types I and II TGF, receptors (12), urokinase type plasminogen activator (uPA) (13), and the human immunodeficiency virus long terminal repeat (HIV-1 LTR) $(13,14)$.

Kruppel-like factors have been implicated in many cellular processes associated with differentiation and development (15). To explore whether KLF6 might behave as an altered tumor suppressor gene, we initially screened for mutations of the KLF6 gene by polymerase chain reaction-single strand conformation polymorphism (PCR-SSCP) in various cancer cell lines, brain and lung tumor samples, matched normal lung samples, as well as a large collection of normal peripheral blood DNAs. Novel missense nucleotide changes occurred in 6 of 88 brain tumors; and gliomas often had low expression of KLF6 compared to normal brain samples. We constructed expression vectors of the mutant and wild-type KLF6 genes and demonstrated that several of the genetic alterations affected the function of the KLF6 protein. Surprisingly, we also found that $45 \%$ of brain tumors and $10 \%$ of normal blood samples had nucleotide changes at $-4,-5$, and -6 upstream of the start site of translation. The functional implication of these changes is unclear.

\section{Materials and methods}

Patient samples and cell lines. Mutational analysis was performed on DNA from both tumor and normal lung tissue of 36 patients diagnosed with non-small cell lung cancers (NSCLC), 89 brain tumor samples including 32 glioblastoma multiforme (GBM), 19 meningiomas, 14 astrocytomas, 6 oligodendrogliomas (or mixed astrocytomas and oligodendrogliomas), and 18 other brain tumor types (schwannomas, craniopharyngiomas, hemangiomas, ependymomas, chordomas, medulloblastoma), as well as 46 cancer cell lines including those established from 13 leukemias, 7 colorectal cancers, 6 non-small cell lung cancers, 5 breast cancers, 5 cervical cancers, 5 lymphomas, 2 sarcomas, 1 vulvar cancer, 1 ovarian cancer and 1 retinoblastoma (name of cell lines provided on request). Written informed consent for research use of tumor tissue was obtained from each patient prior to surgery, according to a protocol approved by the institutional ethics committee. DNA was also extracted from peripheral blood of 144 healthy individuals and used as controls in the experiments.

Polymerase chain reaction-single strand conformation polymorphism. The four exons of the KLF6 gene were amplified from the DNA of each of the samples by PCR. The following sets of primers were designed using GenBank AC023903. Exon 1: 5' cgggctgggtccaac and 5' ggccggctgcgtttac; exon 2a: $5^{\prime}$ cgtgccttctctctggttcatt and 5 ' ctcaattttccegagctgac; exon $2 \mathrm{~b}$ :
5' ccacggccaagtttacetc and 5' ccgcgtgggcactg; exon 3: 5' gcttgtcetgtgctctctcc and 5' cttgcccagcattgtcct; exon 4; 5' gtgct tctgatttgccettg and 5 ' ctcagcctggaagcetttta. Each PCR reaction contained $20 \mathrm{ng}$ of DNA, $10 \mathrm{pmol}$ of each primer, $0.3 \mathrm{mmol}$ of each dNTP, 0.5 units of Platinum Taq DNA Polymerase (Invitrogen, Carlsbad, CA), $2 \mu 1$ of 10X PCR buffer containing $\mathrm{Mg}$ and $3 \mu \mathrm{Ci}$ of dCTP in a total volume of $20 \mu \mathrm{l}$. Thermal cycler parameters were: an initial denaturing step at $94^{\circ} \mathrm{C}$ for 3 min followed by 39 cycles of $94^{\circ} \mathrm{C}$ for $30 \mathrm{sec}, 60^{\circ} \mathrm{C}$ for 30 sec, and $72^{\circ} \mathrm{C}$ for $30 \mathrm{sec}$. Each amplified PCR product $(1 \mu \mathrm{l})$ was diluted to $10 \mu \mathrm{l}$ with denaturing loading dye $(20 \mathrm{mM}$ EDTA, $94 \%$ formamide and $0.05 \%$ of bromophenol blue and xylene cyanol). After denaturing at $95^{\circ} \mathrm{C}$ for $5 \mathrm{~min}, 2 \mu \mathrm{l}$ of each DNA sample was separated on a mutation detection enhancement gel (MDE, Bioproducts, Rockland, ME) at 6W for $20 \mathrm{~h}$. After electrophoresis, gels were dried and exposed to X-OMAT film (Kodak, Rochester, NY).

Sequencing of single strand DNA. Shifted bands and corresponding control bands were excised from the SSCP gel, eluted in TE buffer and reamplified. The amplified DNA was purified using Sigma Spin postreaction purification columns (Sigma, St. Louis, MO). The resulting material was sequenced directly using the Big Dye sequencing kit (PE Applied Biosystems, Foster City, CA) and an automated sequencer (ABI 377 PRISM). The significance of the nucleotide alterations were further studied using Blast 2 sequences (http://www.ncbi.nlm.nih.gov/gorf/bl2.html) with GenBank accession no. AF001461 used as the normal sequence of the KLF6 gene.

RNA isolation and reverse transcription PCR. Extraction of total RNA used Trizol reagent (Invitrogen) according to the manufacturer's instructions. RNA $(2 \mu \mathrm{g})$ was reverse-transcribed with random primers (Invitrogen) and Superscript ${ }^{\mathrm{TM}}$ II reverse transcriptase (Invitrogen) in $20 \mu 1$ reaction volume. The cDNA was used for real-time PCR.

\section{Real-time RT-PCR for KLF6 expression in brain tumors}

Primers and probes. Primers and probe of the KLF6 gene for real-time PCR were designed employing software Primer3 (http://www-genome.wi.mit.edu/cgibin/primer/ primer3www.cgi) according to the nucleotide sequences of GenBank accession no. AF001461: Forward primer, 5' cacga gaccggctacttctc (in exon 1); reverse primer, 5' ccacagatcttcc tggctgt (in exon 2); probe, 5' caacagacctgcctagagctggaacg. Real-time PCR was performed in reaction buffer TaqMan Universal PCR master mix, $100 \mathrm{nM}$ probe and $300 \mathrm{nM}$ primers in a total volume of $25 \mu \mathrm{l}$. After a denaturation step of $10 \mathrm{~min}$ at $95^{\circ} \mathrm{C}$, two-step amplification conditions were 50 cycles of $15 \mathrm{sec}$ at $95^{\circ} \mathrm{C}, 60 \mathrm{sec}$ at $60^{\circ} \mathrm{C}$. Amplification and product detection were performed with an $\mathrm{iCycler}^{\mathrm{iQ}} \mathrm{T}^{\mathrm{TM}}$ system (BioRad, Hercules, CA). We used the B-actin mRNA levels to normalize the expression data of KLF6. Primers and probe of ß-actin were: 5' TCCCTGGAGAAGAGCTACGA (forward), 5' AGGAAGGAAGGCTGGAAGAG (reverse), 5' CAATGA GCGGTTCCGCTGCC (probe). All experiments were done in triplicate. The variance of threshold cycle value between the triplicates was $<5 \%$. The target gene level was divided by the endogenous reference levels to obtain a normalized target 
A
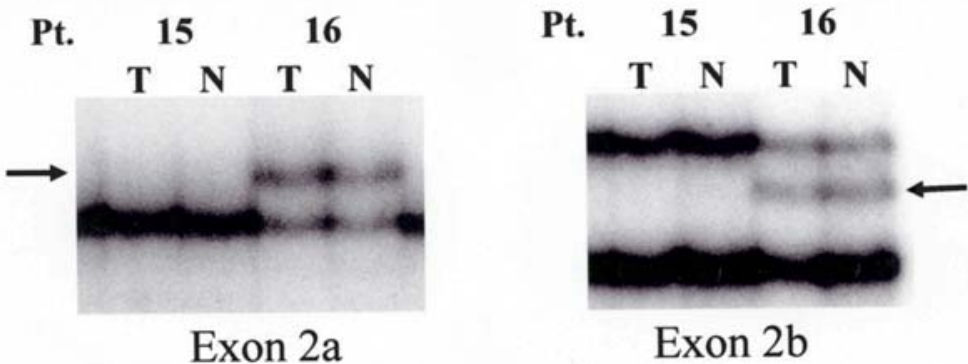

B

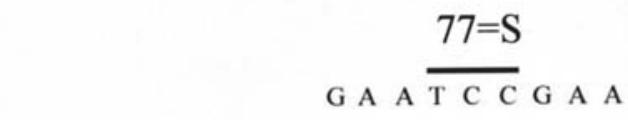

normal control
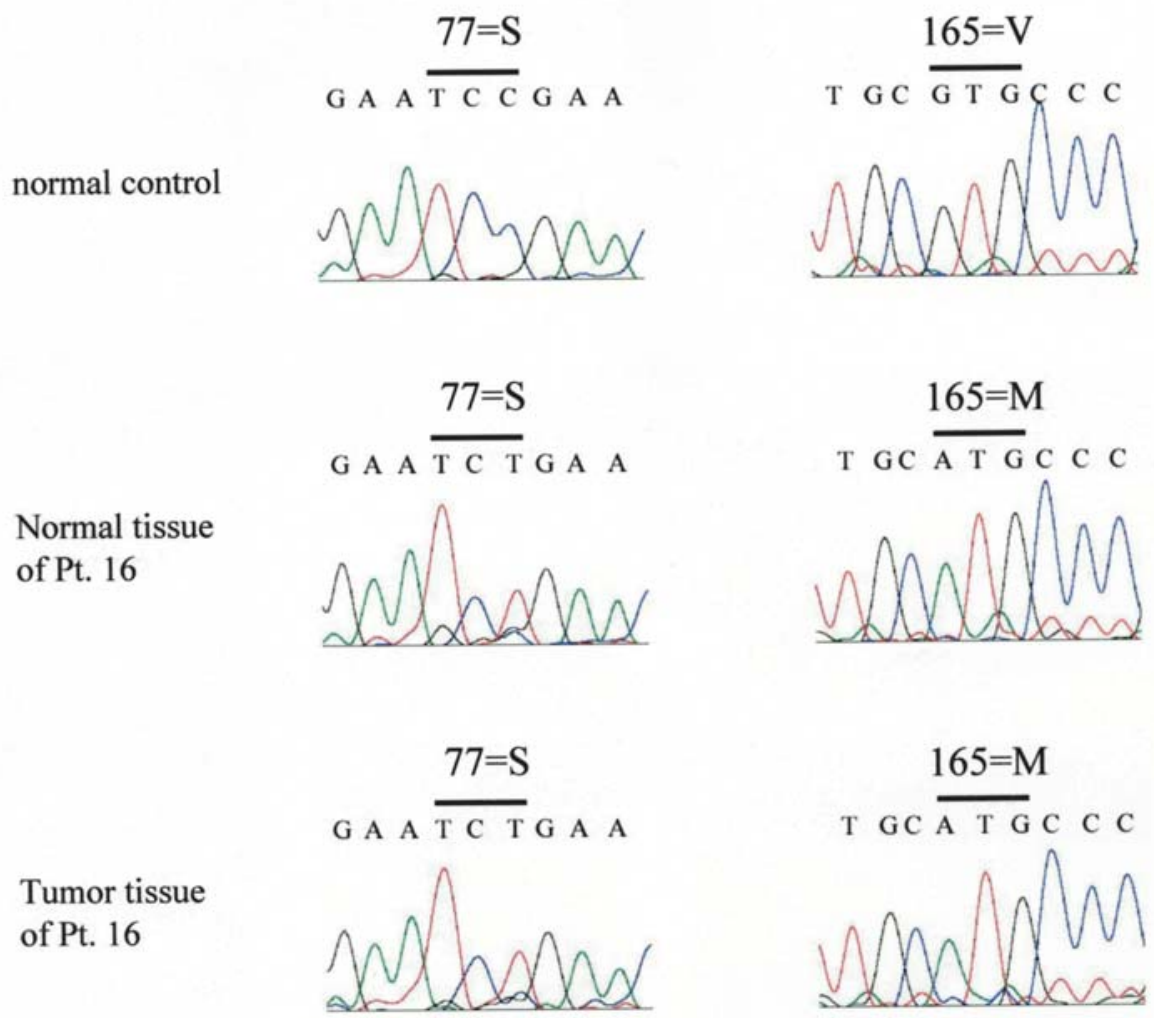

Figure 1. Detection of alterations of the KLF6 gene. Panel A, polymerase chain reaction-single strand conformation polymorphism (PCR-SSCP) analysis of exons $2 \mathrm{a}$ (left panel) and $2 \mathrm{~b}$ (right panel) of the KLF6 gene in lung cancers and their matched normal lung samples. DNA from these samples was amplified with primer sets for 'exon 2a' and 'exon 2b' by PCR yielding fragments of $348 \mathrm{bp}$ and $340 \mathrm{bp}$, respectively. Amplified products were separated on an MDE gel at $6 \mathrm{~W}$ for $\sim 20 \mathrm{~h}$. Each patient (\#15,\#16) had paired samples derived from tumor (T) and normal (N) lung tissue. Arrows indicate the shifted bands. Panel $\mathrm{B}$, sequence trace images showing alterations of the KLF6 gene in tumor and normal lung tissue from patient \#16. The tracings from a normal control, encoding Serine at codon 77 and Valine at codon 165, are shown in the top two panels. The middle and lower two panels show the tracings from normal and tumor lung tissue of patient \#16, showing alterations of the genetic code at codon 165 ( $\mathrm{G}$ to A transition, replaced a Valine with a Methionine) and codon 77 (C to $\mathrm{T}$ transition, resulting in no coding change).

value. The relative expression of target gene was also normalized to a calibrator (mean value). We selected the mean value of normal brain samples as a calibrator. As the final results, the relative expression of each sample was shown as $\mathrm{N}$-fold difference to the calibrator.

Standard curve construction. The standard curve was constructed with 10-fold serially diluted KLF6 cDNA extracted from the U87 cells. A strong linear relationship between the $\mathrm{Ct}$ and the log of the starting copy number was always demonstrated. The standard curves for $\beta$-actin were also constructed (data not shown).

Expression of KLF6 with -4 polymorphism in vivo and vitro. The KLF6 cDNA with either wild-type -4 nucleotide
(Cytosine, C) or polymorphic -4 nucleotide (Adenine, A) was cloned into the expression vector pTracer (Invitrogen, Carlsbad, CA) with V5 tag (Tracer-KLF6-C, Tracer-KLF6-A, respectively). These KLF6 expression vectors were transfected into COS-1 cells for $48 \mathrm{~h}$. The expression of KLF6 (under $\mathrm{P}_{\mathrm{EF}-1 \alpha}$ promoter) was detected by Western blotting (anti-V5 monoclonal antibody from Invitrogen). The expression of GFP protein (under another promoter, PCMV on same vector) was used as an internal control (anti-GFP antibody from Santa Cruz). The expression of KLF6 was also examined in vitro by TNT T7 coupled reticulocyte lysate system (Promega, Madison, WI). The same amount of the two KLF6 expression constructs was used following the protocol of the manual, and the translation of the protein was detected by Western blotting. 
Table I. KLF6: nucleotide changes in cancers.

\begin{tabular}{|c|c|c|c|c|}
\hline Codon number & Nucleotide change & Amino acid change & Sample number & Cell type \\
\hline & & & & Brain tumors ${ }^{a}$ \\
\hline 2 & $\mathrm{gac} \rightarrow \mathrm{ggc}$ & Asp $\rightarrow$ Gly & 120 & Meningioma \\
\hline 6 & atg $\rightarrow$ gtg & Met $\rightarrow$ Val & 317 & Astrocytoma \\
\hline $22^{\mathrm{f}}$ & $\mathrm{ttc} \rightarrow \mathrm{ttt}$ & Phe $\rightarrow$ Phe & 312 & Craniopharyngioma \\
\hline 30 & gag $\rightarrow$ ggg & Glu $\rightarrow$ Gly & 207 & GBM \\
\hline 92 & agc $\rightarrow$ aga & Ser $\rightarrow$ Arg & 114 & Meningioma \\
\hline $165^{\mathrm{e}}$ & gtg $\rightarrow$ atg & $\mathrm{Val} \rightarrow \mathrm{Met}$ & 308 & Meningioma \\
\hline 183 & $\mathrm{ccc} \rightarrow \mathrm{ctc}$ & Pro $\rightarrow$ Ile & 114 & Meningioma \\
\hline $201^{\text {ef }}$ & $\operatorname{cgg} \rightarrow \operatorname{cga}$ & Arg $\rightarrow$ Arg & 315 & Meningioma \\
\hline 276 & $\operatorname{ggc} \rightarrow \operatorname{gcc}$ & Gly $\rightarrow$ Ala & 319 & Craniopharyngioma \\
\hline
\end{tabular}

Squamous cell lung cancer and matched normal samples ${ }^{\text {b }}$

$\begin{array}{cccc}77^{\mathrm{ef}} & \mathrm{tcc} \rightarrow \mathrm{tct} & \mathrm{Ser} \rightarrow \text { Ser } & 16 \\ 165^{\mathrm{e}} & \mathrm{gtg} \rightarrow \text { atg } & \text { Val } \rightarrow \text { Met } & 16 \\ 201^{\mathrm{ef}} & \mathrm{cgg} \rightarrow \mathrm{cga} & \text { Arg } \rightarrow \text { Arg } & 34\end{array}$

\begin{tabular}{|c|c|c|c|c|}
\hline & $\mathrm{tcc} \rightarrow \mathrm{tct}$ & $\mathrm{Ser} \rightarrow \mathrm{Ser}$ & DAUDI & $\begin{array}{l}\text { Cancer cell line }^{c} \\
\text { Burkitts lymphoma }\end{array}$ \\
\hline $165^{\mathrm{e}}$ & gtg $\rightarrow$ atg & $\mathrm{Val} \rightarrow \mathrm{Met}$ & DAUDI & Burkitts lymphoma \\
\hline
\end{tabular}

\begin{tabular}{l}
\hline- \\
\hline a Total of 89 brain tumor samples. ${ }^{\mathrm{b}}$ Total of 36 lung caners and their normal matched samples (KLF6 sequences identical in both). ${ }^{\mathrm{c}}$ Total of \\
46 cancer cell lines (see Material and methods for their tissue type). ${ }^{\mathrm{d}}$ Total of 144 samples of white blood cells (WBC) from normal \\
individuals. ${ }^{\mathrm{e} P o l y m o r p h i s m,}{ }^{\mathrm{f}}$ silent nucleotide change.
\end{tabular}

Western blot analysis. Cells were harvested for total cell lysates with RIPA buffer (1\% Nonidet P-40, $0.5 \%$ sodium deoxycholate, $0.1 \% \mathrm{SDS}, 50 \mathrm{mM}$ Tris-HCl, $\mathrm{pH} 7.5$ ) containing a protease inhibitor mixture (Roche Diagnostics $\mathrm{GmbH}$, Mannheim, Germany), as well as $1 \mathrm{mM} \mathrm{NaF}$ and $1 \mathrm{mM} \mathrm{NaVO}$. Cell lysates were centrifuged at 13,000 rpm for $10 \mathrm{~min}$ at $4^{\circ} \mathrm{C}$. The supernatant was collected, and the protein concentration was measured. The lysates $(30 \mu \mathrm{g})$ were denatured in sample buffer (10\% glycerol, $5 \%$ Bmercaptoethanol, 2.3\% SDS, $62.5 \mathrm{mM}$ Tris-HCl, $\mathrm{pH}$ 6.8) by boiling and then subjected to $4-15 \%$ SDS-PAGE followed by electro-transfer to polyvinylidene difluoride membrane. After antibody hybridization, the immune complex was visualized with Supersignal West Pico chemiluminescent substrate (Pierce, Rockford, IL).

KLF6 mutant constructs. Full-length KLF6 cloned into pTracer vector was used for site-directed mutagenesis; pTracer-wildtype KLF6 was mutated using the Quick-Change site directed mutagenesis kit (Stratagene). All constructs were sequenced to confirm mutation of the appropriate base pair.

Cell growth assay. Either KLF6 (wild-type or mutant) expression pTracer vector or empty pTracer vector (control) and anti-puromycin vector were co-transfected into the same number of T98G (GBM) cells. After selection with $8 \mu \mathrm{g} / \mathrm{ml}$ of puromycin for $18 \mathrm{~h}$, cells were cultured in puromycin-free medium for an additional 5 days. The number of cells was measured by MTT assay.

\section{Results}

Mutational analysis of KLF6 gene. The following was analyzed for mutations of the KLF6 gene by PCR-SSCP: 89 brain tumors and 7 normal brain samples, 36 lung cancers and their matched normal lung, 46 cancer cell lines, and 144 normal peripheral blood samples. Aberrantly migrating bands on SSCP were detected in the coding region of KLF6 in 9 of $89(10 \%)$ brain tumors, 3 of $36(8 \%)$ lung cancer samples, and 2 of 46 (4\%) cancer cell lines (Fig. 1A). Sequencing of the shifted bands showed nucleotide substitutions in the coding region of KLF6, most of which resulted in amino acid changes including 7 of $89(8 \%)$ brain tumors, and 1 of $36(3 \%)$ lung cancers, 1 of 46 cancer cell lines (Fig. 1 B; Table I). A nucleotide change at either codon 77, 165 or 201 occurred in more than one sample. In our collection of lung cancers, we always studied a matched normal and tumor sample from the same individual. In each of the 3 cases with a nucleotide 
Table II. KLF6 nucleotide changes occurring 5' to start site of translation.

\begin{tabular}{lcccc}
\hline & $-4 \mathrm{C}>\mathrm{A}$ & $-4 \mathrm{C}>\mathrm{T}$ & $-5 \mathrm{C}>\mathrm{T}$ & $-6 \mathrm{C}>\mathrm{T}$ \\
\hline Brain tumor & $20 / 89(23 \%)$ & $5 / 89(6 \%)$ & $11 / 89(12 \%)$ & $4 / 89(5 \%)$ \\
Normal blood & $7 / 144(5 \%)$ & $2 / 144(1 \%)$ & $2 / 144(1 \%)$ & $3 / 144(2 \%)$ \\
\hline
\end{tabular}

$-4 \mathrm{C}>\mathrm{A}$, implies the Cytosine is replaced by Adenine at position -4 from the start site of translation (other nucleotide changes follow similar format); numerator, the number of samples with the nucleotide change; denominator, the total number of examined samples.

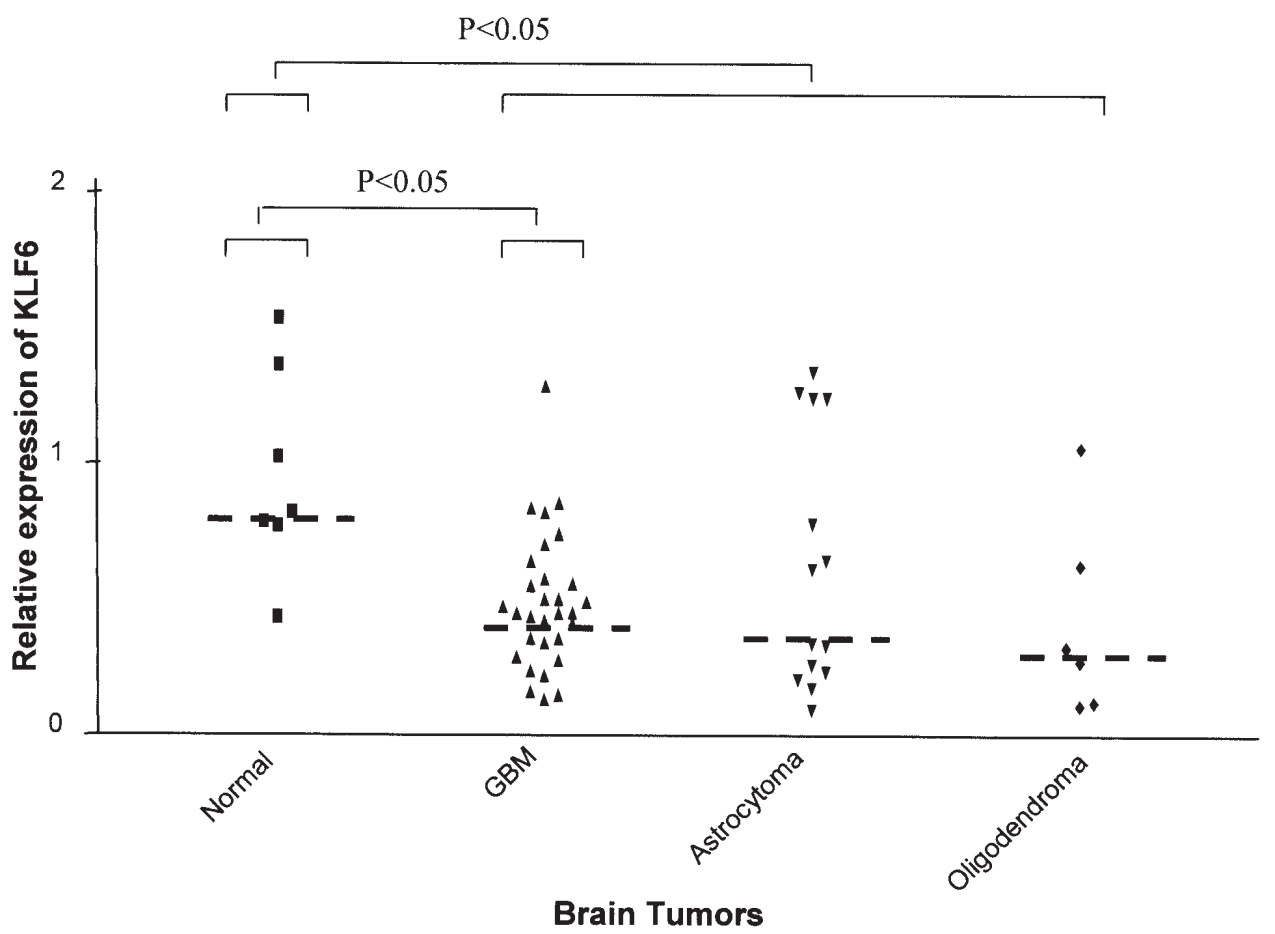

Figure 2. Quantification of mRNA expression of the KLF6 gene using real-time RT-PCR. The relative mRNA expression level of KLF6 in gliomas and normal brain tissues was quantified by real-time RT-PCR. Values of all samples represent the mean of 3 separate measurements. The variance of threshold cycle values between the triplicates was $<5 \%$. The values of copy number of KLF6 cDNA were used to indicate relative mRNA expression of KLF6. The copy number of KLF6 cDNA in brain tissue samples was calculated from the KLF6 standard curve of real-time PCR and normalized to the copy number of B-actin in each of the samples. Dash lines are the median values of the KLF6 expression for each group. GBM, glioblastoma multiforme. Statistics (t-test) showed $\mathrm{p}<0.05$ for normal brain samples compared to either GBM or all glioma samples.

change, the normal sample had the same alteration as was found in the tumors (Fig. 1, Table I). Eliminating the previously unreported polymorphism, 7 of $89(8 \%)$ of brain tumors had missense changes of the KLF6 gene. These included 4 of 19 meningiomas, 1 of 32 GBM, 1 of 3 craniopharyngioma, and 1 of 14 astrocytomas.

To our surprise, we found 3 sites of frequent polymorphic substitutions at either $-6,-5$, or -4 nucleotide upstream of the start site of translation of the KLF6 gene ( $-6 \mathrm{C}>\mathrm{T},-5 \mathrm{C}>\mathrm{T}$, $-4 \mathrm{C}>\mathrm{T},-4 \mathrm{C}>\mathrm{A}$ ) in the samples (Table II). The frequency of nucleotide substitution at one of these sites was statistically different $(\mathrm{p}<0.05)$ between brain cancer $40 / 89(45 \%)$ and normal peripheral blood 14/144 (10\%). The site with the highest frequency of polymorphism was -4 C>A: $23 \%(20 / 89)$ in brain tumors, and 5\% (7/144) in normal peripheral blood.

Expression level of KLF6 mRNA in normal brain and brain tumors. Tumor suppressor genes can be lost in a cancer by mutating, deleting or silencing the gene (e.g., methylation of its promoter). Real-time RT-PCR was performed to measure the expression of KLF6 in 7 normal brain samples and 50 gliomas (30 GBM, 14 astrocytomas, 6 oligodendromas). Levels of KLF6 were significantly higher in normal brain samples compared to gliomas including GBMs ( $\mathrm{p}<0.05$, Fig. 2).

Effect of genetic alterations of KLF6 on the proliferation of GBM cells. Tumor suppressor genes often behave as brakes to unopposed cellular proliferation. Thus, the protein coded for by the tumor suppressor genes usually slows cellular proliferation. The cancer frequently mutates this gene so that the resulting protein no longer suppresses growth. We made six KLF6 expression constructs. Four of them represent missense alterations that we detected in the brain tumors; the fifth was a deletion of exon 3 which occurs by alternative splicing; and the sixth was the wild-type KLF6. Each construct was sequenced and also confirmed to be the correct molecular 


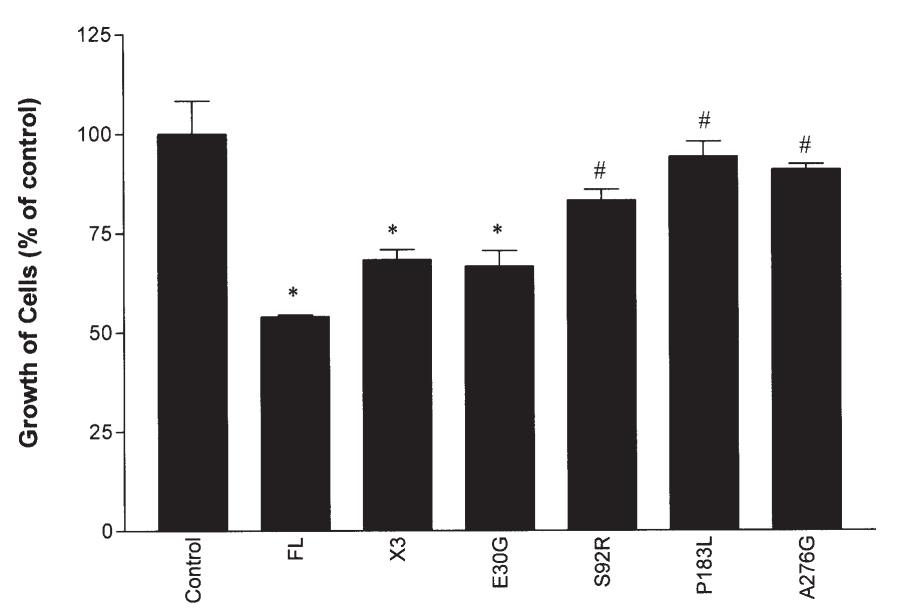

Figure 3. KLF6 effect on the growth of GBM cells. T98G GBM cells were co-transfected with either the indicated mutant or wild-type KLF6 constructs and the puromycin-resistant vector. Empty pTracer vector was used as control. After selection with $8 \mu \mathrm{g} / \mathrm{ml}$ of puromycin for $18 \mathrm{~h}$ to kill cells not receiving the resistant vector, the cells were cultured in puromycin-free medium for an additional 5 days. The number of cells was measured by MTT assay. Results represent mean \pm SD of 3 experiments with triplicate wells per experimental point $\left({ }^{*} \mathrm{p}<0.05\right.$, compared to control; ${ }^{*} \mathrm{p}<0.05$, compared to FL KLF6). FL, full length cDNA of KLF6; X3, exon 3 deletion of KLF6; E30G, KLF6 with Glu changed to Gly at codon 30; S92R, Ser changed to Arg at codon 92; P183L, Pro changed to Leu at codon 183; A276G, Ala changed to Gly at codon 276.

weight by Western blot of lysates from 293 cells transiently transfected with the expression vector (data not shown). The ability of these KLF6 expression vectors to affect cell growth was measured by MTT. The wild-type, exon 3 deletion, and E30G mutant KLF6 reduced the growth of the T98G GBM cells $(\mathrm{p}<0.05)$. The S92R, P183L and A276G mutant LKF6 had no statistically significant effect on the growth of the GBM cells (Fig. 3).

Effect of -4 nucleotide on the expression of KLF6. Because the nucleotide substitutions at $-6,-5$, or -4 upstream of the start site of translation of KLF6 gene are near the Kozak conserved sequences, we wondered if these changes could affect translation of the protein. As $-4 \mathrm{C}>\mathrm{A}$ polymorphism was the most frequent change in brain tumors (23\%), we chose to investigate the effect of this polymorphism on the expression of KLF6 in vivo and in vitro. pTracer vector was used in our experiments. It is a vector with two promoters; one promoter was used to express $K L F 6$ and another for GFP as an internal control. The Tracer-KLF6-C (contains KLF6 cDNA with C at -4 from the start site of translation), and Tracer-KLF6-A (contains KLF6 cDNA with A at -4 from the start site of translation). The expression vectors were transfected into cells for $48 \mathrm{~h}$. The levels of protein expression of KLF6 and GFP were examined by Western blotting; the $-4 \mathrm{C}>\mathrm{A}$ polymorphism did not affect the expression of KLF6 in vivo (Fig. 4A). Expression of GFP was comparable in the two experimental lanes. In addition, real-time RT-PCR did not show a significant difference of mRNA levels of KLF6 between the brain tumor samples with -4 wild-type (C) and the -4 polymorphism (A) (data not shown). In vitro, the efficiency of translation of the KLF6 gene was also measured using the T7 translation system in vitro; no differences occurred between the levels of
A

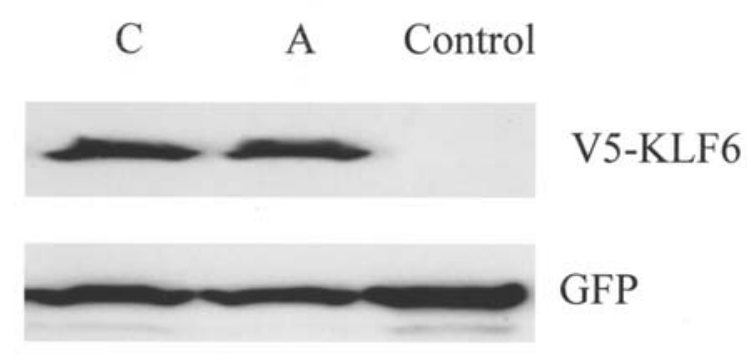

B

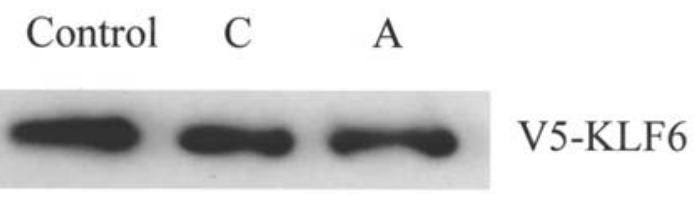

Figure 4. Polymorphism at $-4(-4 \mathrm{C}>\mathrm{A})$ of KLF6 and its affect on KLF6 expression. Panel A, KLF6 cDNA with high frequency nucleotide type (-4 Cytosine, C) and low frequency nucleotide type (-4 Adenine, A) were cloned into the expression vector pTracer (Tracer-KLF6-C, Tracer-KLF6-A), with a V5 tag on the C terminus. GFP cDNA controlled by a separate promoter in pTracer, was used as an internal control. These expression vectors were transfected into Cos-1 cells. After $48 \mathrm{~h}$, cells were harvested and lysates were run on Western blot. Cos-1 cells were transfected with pTracer vector not having KLF6 but containing GFP as control. KLF6 and GFP proteins were detected by V5 and GFP antibodies, respectively. Panel B, the same amount of pTracer-KLF6-C and pTracer-KLF6-A were used for in vitro transcription and translation using the TNT T7 coupled reticulocyte lysate system. The KLF6 product was subjected to Western blot analysis. Cell lysate from Cos-1 cells transfected with Tracer-KLF6-C was used as positive control. Blots were probed with V5 antibody.

expression of $-4 \mathrm{C}$ and $-4 \mathrm{~A}$ KLF6 (Fig. 4B). These results suggest that the -4 polymorphism $(-4 \mathrm{C}>\mathrm{A})$ has no detectable effect on KLF6 expression.

\section{Discussion}

KLF6 is a ubiquitously expressed Kruppel-like transcription factor that is regarded as a tumor suppressor. An initial study suggested that $55 \%$ of a subset of human prostate cancers had KLF6 mutations with a loss of the normal allele. Their functional analysis revealed that wild-type KLF6 up-regulated p $21^{\text {wafl }}$ in a p53-independent manner and significantly reduced cell proliferation; in contrast, tumor-derived mutant forms of KLF6 neither increased p21 nor decreased proliferation (1). Another study reported that 3 different mutations (Glu75Val, Ser136Arg, Arg243Lys) were found in 19 sporadic nasopharyngeal carcinomas $(3 / 19,16 \%)$, suggesting that KLF6 might be involved in carcinogenesis of sporadic nasopharyngeal carcinomas (2). The KLF6 gene was reported to be mutated in $8 \%(13 / 155)$ of gliomas, suggesting that mutations of the KLF6 gene plays a role in the pathogenesis of this cancer (3). Also, studies have suggested an association between KLF6 function and tumorigenesis (4-6,16-25). In contrast, other investigations have suggested that KLF6 mutations were infrequent in cancers of the prostate, liver and colon (7-9). Some investigators have conjectured that these mutations may be artifacts because the DNAs were 
extracted from formalin-fixed paraffin-embedded tissue (9). All of our DNA was from either fresh or fresh-frozen samples.

We analyzed 36 matched lung cancers, 89 brain tumor samples, 46 miscellaneous human cell lines from various cancers and 144 normal white blood cell samples by PCRSSCP for mutations/polymorphisms of the KLF6 gene within the coding region. Alterations were detected in 3 of 36 lung cancers $(8 \%), 9$ of 89 brain tumors (10\%), and 2 of 46 cancer cell lines $(4 \%)$, and 0/144 normal samples. Of note, lung cancer sample \#16 and cell line DAUDI had the same double mutation: silent $\mathrm{C}$ to $\mathrm{T}$ transition at codon 77 , and $\mathrm{G}$ to $\mathrm{A}$ transition at codon 165 which changed Valine to Methionine. Also, a brain tumor had a double mutation: $\mathrm{C}$ to A transversion at codon 92 (Ser92Arg), and $\mathrm{C}$ to $\mathrm{T}$ transition at codon 183 (Pro183Leu). In the 14 alterations in the coding region, 9 coded for an amino acid substitution. Of further note, most of the amino acid substitutions (8/9) were found in the transactivation domain (AA1-201) of KLF6, similar to that reported for prostate cancer (1). Are these nucleotide changes polymorphism or mutations? The Val165Met and the two silent alterations at codons 77 and 201 are polymorphism because they occurred in both the normal lung and matched cancer samples (matched samples No. 16 and 34). Additional studies showed that of the 46 cancer cell lines, only one had an abnormality (DAUDI, Val to Met), and this change was also observed in normal lung tissue. Thus, neither lung cancer nor a large variety of cancer cell lines had KLF6 mutations.

Of the 89 brain tumors 6 had a nucleotide alteration that resulted in a change in an amino acid of KLF6. Also, these nucleotide changes were not found in 144 normal samples, 46 cancer cell lines, 36 lung cancers or their normal control. In order to explore the functional implication of these nucleotide changes, we constructed expression vectors having the KLF6 missense mutations that we identified in brain tumors (E30G, S92R, P183L, A276G), as well as the exon 3 deletion (alternative spliced product of $K L F 6$ ). Their effect on growth of GBM cells was examined. Wild-type KLF6 significantly reduced growth ( $\sim 50 \%$ reduction) of GBM cells consistent with a functional tumor suppressor gene behaviour. In contrast, the S92R, P183L and A276G mutant KLF6 lost this growth inhibitory ability when expressed in GBM cells, suggesting these three missense mutations resulted in significant loss of tumor suppressor activity of KLF6. Loss of exon 3 and E30G nucleotide change still significantly inhibited growth of GBM cells.

Another finding from this study was that GBM frequently had either a $\mathrm{C}$ to $\mathrm{A}$ change at nucleotide -4 , or a $\mathrm{C}$ to $\mathrm{T}$ change at either nucleotide $-6,-5$, or -4 . One of these changes occurred in 40 of 89 samples (45\%). In contrast, these changes occurred in only 14 of 144 normal samples $(10 \%)$. The difference between tumor and normal is statically significant $(p<0.05)$. Because these nucleotide changes were near the Kozak consensus sequence, we investigated whether these change could affect translation of KLF6. The C to A change at nucleotide -4 was very frequent in brain tumor samples $(20 / 89,23 \%)$ compared to samples from peripheral blood of normal individuals $(7 / 144,5 \%)$; therefore, we focused on this alteration and made expression vectors having either a $\mathrm{C}$ or A at -4 of KLF6. We did not detect a change in efficiency of KLF6 protein expression between these two KLK6 constructs either in cells or in vitro. Therefore, the functional significance of these changes remains to be clarified.

The frequency of nucleotide change of KLF6 was also explored by blast search of human EST sequences in NCBI blast site. Specifically, we investigated codons 2, 6, 22, 30, 92, 165,183 , and 201 (nucleotide changes noted in our studies). No matched sequences were found in almost 130 ESTs as compared to the substitutions observed in our tumor samples.

In summary, expression of KLF6 was lower in brain tumors compared to normal brain samples. Transfection of the wildtype KLF6 into GBM cells slowed their growth. Therefore, decreasing the expression of this gene may give developing brain tumors a growth advantage. Further studies are required to understand how levels of this protein are decreased in brain tumors. We also detected several novel coding variations of the KLF6 gene, occurring in lung tissue (normal and cancer) $(3 \%)$ and brain cancers $(8 \%)$ as well as a cancer cell line $(2 \%)$. Excluding polymorphism that we identified, 6 of $89(7 \%)$ nucleotide changes were probably missense mutations. Of the 4 mutants of these KLFG containing missense mutations that were examined, 3 had significantly less ability to inhibit GBM cell growth compared to the wild-type KLF6. We also noted that alterations of the $-4,-5,-6$ upstream nucleotides of $K L F 6$ occurred in the brain tumor samples with the $\mathrm{C}$ to A change at nucleotide -4 occurring at an especially high frequency $(23 \%$ of brain tumors). This study suggests that either silencing or mutations of KLF6 may be involved in the development or progression of a subset of brain cancers.

\section{Acknowledgements}

This work was supported by the George Harrison Fund, the Inger Foundation, as well as the Parker Hughes Fund. Alexey Chumakov is the recipient of NIH training grant CA75956 (fellowship at UCLA). H. Phillip Koeffler holds the Mark Goodson Endowed Chair of Oncology Research at CSMC, and is a member of the Jonsson Cancer Center and the Molecular Biology Institute of UCLA. This work is dedicated to the memory of Matt Schreck.

\section{References}

1. Narla G, Heath KE, Reeves HL, et al: KLF6, a candidate tumor suppressor gene mutated in prostate cancer. Science 294: 2563-2566, 2001.

2. Chen HK, Liu XQ, Lin J, Chen TY, Feng QS and Zeng YX: Mutation analysis of KLF6 gene in human nasopharyngeal carcinomas. Ai Zheng 21: 1047-1050, 2002.

3. Jeng YM and Hsu HC: KLF6: a putative tumor suppressor gene, is mutated in astrocytic gliomas. Int J Cancer 105: 625-629, 2003.

4. Vax VV, Gueorguiev M, Dedov II, Grossman AB and Korbonits M: The Kruppel-like transcription factor 6 gene in sporadic pituitary tumours. Endocr Relat Cancer 10: 397-402, 2003.

5. Ito G, Uchiyama M, Kondo M, et al: Kruppel-like factor 6 is frequently down-regulated and induces apoptosis in non-small cell lung cancer cells. Cancer Res 64: 3838-3843, 2004.

6. Reeves HL, Narla G, Ogunbiyi O, et al: Kruppel-like factor 6 (KLF6) is a tumor-suppressor gene frequently inactivated in colorectal cancer. Gastroenterology 126: 1090-1103, 2004.

7. Koivisto PA, Hyytinen ER, Matikainen M, Tammela TL, Ikonen T and Schleutker J: Kruppel-like factor 6 germ-line mutations are infrequent in Finnish hereditary prostate cancer. J Urol 172: 506-507, 2004. 
8. Boyault S, Herault A, Balabaud C and Zucman-Rossi J: Absence of KLF6 gene mutation in 71 hepatocellular carcinomas. Hepatology 41: 681-682, 2005.

9. Lievre A, Landi B, Cote JF, et al: Absence of mutation in the putative tumor-suppressor gene KLF6 in colorectal cancers. Oncogene 24: 7253-7256, 2005.

10. Koritschoner NP, Bocco JL, GM, Dumur CI, Flury A and Patrito LC: A novel human zinc finger protein that interacts with the core promoter element of a TATA box-less gene. J Biol Chem 272: 9573-9580, 1997.

11. Ratziu V, Lalazar A, Wong L, et al: Zf9, a Kruppel-like transcription factor up-regulated in vivo during early hepatic fibrosis. Proc Natl Acad Sci USA 95: 9500-9505, 1998.

12. Kim Y, Ratziu V, Choi SG, et al: Transcriptional activation of transforming growth factor beta 1 and its receptors by the Kruppel-like factor Zf9/core promoter-binding protein and Sp1. Potential mechanisms for autocrine fibrogenesis in response to injury. J Biol Chem 273: 33750-33758, 1998.

13. Kojima S, Hayashi S, Shimokado K, et al: Transcriptional activation of urokinase by the Kruppel-like factor Zf9/COPEB activates latent TGF-beta1 in vascular endothelial cells. Blood 95: 1309-1316, 2000 .

14. Suzuki T, Yamamoto T, Kurabayashi M, Nagai R, Yazaki Y and Horikoshi M: Isolation and initial characterization of GBF, a novel DNA-binding zinc finger protein that binds to the GCrich binding sites of the HIV-1 promoter. J Biochem (Tokyo) 124: 389-395, 1998.

15. Bieker JJ: Kruppel-like factors: three fingers in many pies. J Biol Chem 276: 34355-34358, 2001.

16. Benzeno S, Narla G, Allina J, et al: Cyclin-dependent kinase inhibition by the KLF6 tumor suppressor protein through interaction with cyclin D1. Cancer Res 64: 3885-3891, 2004.
17. Cho YG, Kim CJ, Park CH, et al: Genetic alterations of the KLF6 gene in gastric cancer. Oncogene 24: 4588-4590, 2005.

18. Kimmelman AC, Qiao RF, Narla G, et al: Suppression of glioblastoma tumorigenicity by the Kruppel-like transcription factor KLF6. Oncogene 23: 5077-8503, 2004.

19. Kremer-Tal S, Reeves HL, Narla G, et al: Frequent inactivation of the tumor suppressor Kruppel-like factor 6 (KLF6) in hepatocellular carcinoma. Hepatology 40: 1047-1052, 2004.

20. Narla G, DiFeo A, Reeves HL, et al: A germline DNA polymorphism enhances alternative splicing of the KLF6 tumor suppressor gene and is associated with increased prostate cancer risk. Cancer Res 65: 1213-1222, 2005.

21. Rubinstein M, Idelman G, Plymate SR, Narla G, Friedman SL and Werner H: Transcriptional activation of the insulin-like growth factor I receptor gene by the Kruppel-like factor 6 (KLF6) tumor suppressor protein: potential interactions between KLF6 and p53. Endocrinology 145: 3769-3777, 2004.

22. Wang S, Chen X, Zhang W and Qiu F: KLF6mRNA expression in primary hepatocellular carcinoma. J Huazhong Univ Sci Technolog Med Sci 24: 585-587, 2004.

23. Wang SP, Chen XP and Qiu FZ: A candidate tumor suppressor gene mutated in primary hepatocellular carcinoma: kruppel-like factor 6. Zhonghua Wai Ke Za Zhi 42: 1258-1261, 2004.

24. Kohler B, Wolter M, Blaschke B and Reifenberger G: Absence of mutations in the putative tumor suppressor gene KLF6 in glioblastomas and meningiomas. Int J Cancer 111: 644-645, 2004.

25. Koivisto PA, Zhang X, Sallinen SL, et al: Absence of KLF6 gene mutations in human astrocytic tumors and cell lines. Int $\mathbf{J}$ Cancer 111: 642-643, 2004. 